\title{
Lorenz partial order: the best known logical framework to define evenness indices
}

\author{
F. Gosselin
}

\begin{abstract}
Cemagref, URE Ecosystèmes Forestiers et Paysages, Domaine des Barres, 45290 Nogent-sur-Vernisson, France. Fax: 00332389503 44,E-mail:frederic.gosselin@cemagref.fr
\end{abstract}

Keywords: Biodiversity, Equitability, Lorenz partial order, Regularity.

\begin{abstract}
Evenness is generally regarded as the constituent of species diversity which is independent of species richness. The group of most popular evenness indices has recently changed, following the influential work of Smith and Wilson (1996). My first point is to argue that this work failed to define coherently what evenness should be and thus advocated the use of indices of different nature. Instead, I propose to use the Lorenz partial order to choose indices that have a behaviour compatible with it. I then suggest a list of such evenness indices and build four series of "new" evenness indices. Finally, I discuss the interest of the Lorenz partial order and of the various evenness indices that are compatible with it.
\end{abstract}

"Before measurements can be meaningful they must be directed to the right things and, even in science, finding these things is the major achievement: entitation is more important than quantitation." Gerard (1965) in McIntosh (1967)

\section{Introduction}

Evenness (or equitability) is generally regarded as the constituent of species diversity which is independent of species richness. It is embedded in some hypotheses, theories or models relating diversity to other community parameters (e.g., Drobner et al. 1998, Weiher and Keddy 1999), ecological parameters (Wilson et al. 1999) or ecosystem functioning (Nijs and Roy 2000, Wilsey and Potvin 2000). A recent work by Smith and Wilson (1996) reconsidered evenness indices on the basis of their empirical and mathematical properties, and this paper seems influential (cf. Drobner et al. 1998, Weiher and Keddy 1999, Krebs 1999, Wilson et al. 1999). Yet, I believe that Smith and Wilson failed to define evenness clearly, and proposed the use of "evenness" indices that were in fact of varying nature. My first point is therefore to use a clear and simple mathematical framework, the Lorenz partial order, to define evenness consistently. I compare it with Smith and Wilson's (1996) construct, as well as with the study of Engen (1979). This complements the earlier works by Taillie (1979), Nijssen et al. (1998), and Rous- seau et al. (1999) on the subject. In the second part, I review the compliance with the Lorenz partial order of some evenness indices found in the literature. A series of new and simple evenness indices compatible with the Lorenz partial order is also proposed.

\section{The Lorenz partial order and its relationship to properties required for evenness indices}

\section{The Lorenz partial order and Lorenz curves}

In this paper, $p_{i}$ denotes the relative abundance of the $i$-th least abundant species in the community (i.e., $p_{1}$ is for the least abundant species). $S$ is the species richness of the community.

The Lorenz partial order has been developed in economics and applied mathematics (Dalton 1920, Marshall and Olkin 1979) and has been proposed by several authors as the foundation of the ecological notion of evenness (or equitability) (cf. Taillie 1979, Nijssenn et al. 1998, Rousseau et al. 1999). It is an order in that it is reflexive, transitive and antisymmetric, but it is only partial because we cannot order all pairs of communities. In this framework, evenness is a measure of the relative apportionment of abundance among the proportion of the species considered as present in the community. The two main axioms that actualise this statement are: 
- two communities are intrinsically as even (that is, equal with respect to this partial order) if one community is obtained from the other by replicating the community, that is replacing each species in the original community by a fixed number $n$ of species with the same absolute abundance as the original species (this will be called the replication principle);

- a community is intrinsically less even than another community (that is, "less" according to this partial order) if it has the same species number as the other community and it is obtained from the other community by the transfer of a quantity of relative abundance from a species to a more abundant species (this is called the transfer principle).

The Lorenz partial order can be visualized by the use of Lorenz curves: each community is represented by a curve linking the points with the cumulative proportions of species as the abscissa (e.g. the $k$-th less abundant species in a community of $S$ species has abscissa $k / S$ ) and with the ranked cumulative proportions of abundances as ordinates (e.g. the $k$-th less abundant species in a community of $S$ species has ordinate the sum of the relative abundances of the $k$ less abundant species, $\sum_{i=1}^{k} p_{i}$; cf. Fig. 1). Then, a community has a Lorenz curve everywhere above that of another community if and only if it is intrinsically more even than the other community.

The Lorenz partial order and other requirements proposed to define evenness

This clear mathematical construct conflicts with the empirical requirements proposed by Smith and Wilson (1996) for evenness indices; as well as with the mathematical construct by Engen (1979). The discrepancies between the notion of evenness as defined by the Lorenz partial order and the empirical properties required by Smith and Wilson (1996) are listed in Table 1. Three main conflicts are emphasized here:

Symmetry. According to Features 13 and 14 in Smith and Wilson (1996) (cf. Table 1), the evenness of, say the community with four species having relative abundances $(0.01,0.01,0.49,0.49)$ should be higher than that of $(0.01$, $0.01,0.01,0.97)$ and $(0.01,0.33,0.33,0.33)$, those last two communities being of equal evenness. This point is contrary to the transfer principle of the Lorenz partial order, and to the very notion of evenness as being a quantification of the relative apportionment of abundance between the proportion of species present. Features 13 and 14 in Smith and Wilson (1996) therefore seem to rely on a concept of evenness different from that of the Lorenz partial order, which we cannot readily identify.

Minimum evenness independent of species richness. According to Features 8 and 10 in Smith and Wilson (1996), the evenness index of $(0.01,0.01,0.01,0.97)$ should be close to the absolute minimum of the evenness index. This is not required by the Lorenz partial order. As I show now, this is in fact in contradiction with it.

Due to the transfer principle, a community with $S$ species which is clearly intrinsically less even than all other communities with $S$ species is the community composed of one hyperdominant species with relative abundance 1 and $S$-1 species with relative abundance 0 , be it considered as a limit community or as a real community with one species detected and $S$-1 species considered as present but undetected. Yet, it is also clear from the Lorenz curves of these "minimal" evenness communities that the minimally even community with $S$-1 species is intrinsically more even that the minimally even community with species $S^{\prime}>S$ (cf. Fig. 2).
Figure 1. Lorenz curves for absolute abundances $(1,1,1,1$, $10,100)$ of community 1 and $(10,100)$ of community 2 . Since the Lorenz curve of community 2 is everywhere above that of community 1 , community 2 is intrinsically more even than the other according to the Lorenz partial order.

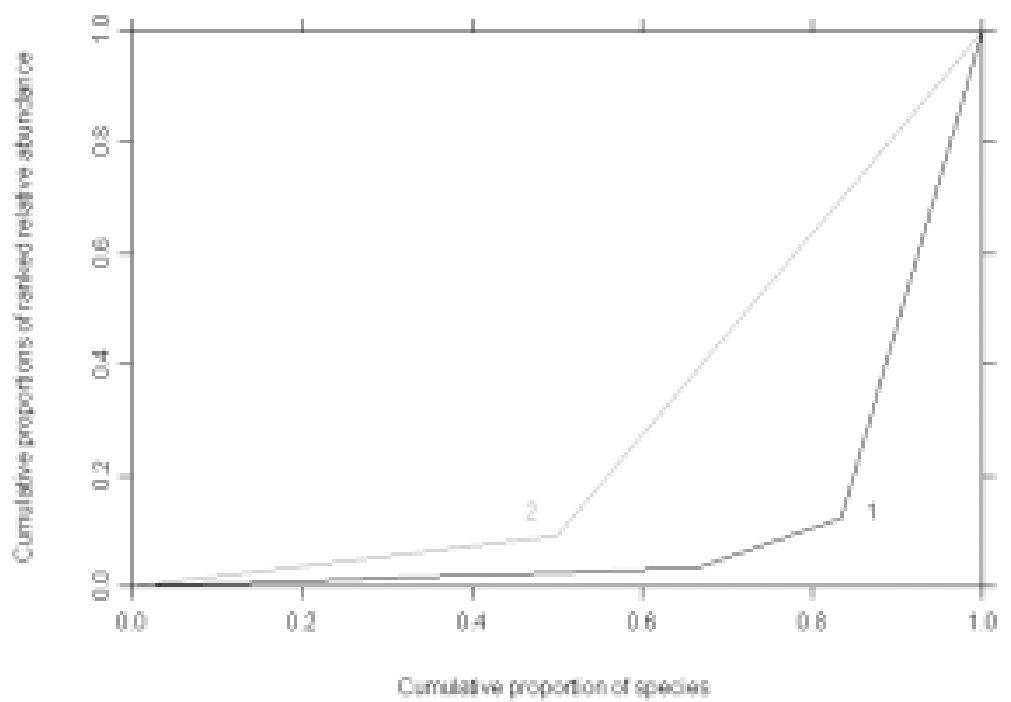


Table 1. List of the requirements and features proposed by Smith and Wilson (1996) to define the various components of evenness, with their agreement, opposition or unrelated character with the Lorenz partial order.

\begin{tabular}{|l|l|}
\hline Requirements and features in Smith and Wilson (1996) & $\begin{array}{l}\text { Agreement, opposite or } \\
\text { unrelated to the Lorenz } \\
\text { partial order }\end{array}$ \\
\hline Requirement 1: independent of species richness & Agreement \\
\hline $\begin{array}{l}\text { Requirement 2: decreased by reducing marginally the abundance of the most } \\
\text { minor species }\end{array}$ & Agreement \\
\hline Requirement 3: decreased by the addition of a very minor species & Agreement \\
\hline Requirement 4: unaffected by the units used & Agreement \\
\hline Feature 5: maximal when species abundances are equal & Agreement \\
\hline Feature 6: maximum value 1 & Unrelated \\
\hline $\begin{array}{l}\text { Feature 7: minimal, for any number of species, when the species abundances } \\
\text { are as unequal as possible }\end{array}$ & Agreement \\
\hline $\begin{array}{l}\text { Feature 8: a value close to its minimum when the community is as uneven as } \\
\text { we would likely to meet }\end{array}$ & Opposite (cf. text) \\
\hline Feature 9: minimum value 0 & Unrelated \\
\hline Feature 10: minimum attainable with any number of species & Opposite (cf. text) \\
\hline $\begin{array}{l}\text { Feature 11: a value in the middle of the scale for communities that we would } \\
\text { intuitively consider intermediate }\end{array}$ & Unrelated \\
\hline $\begin{array}{l}\text { Feature 12: respond in a reasonable way to a series of communities that } \\
\text { intuitively changes in evenness (as interpreted by Smith and Wilson 1996) } \\
\text { intuitively changes in evenness (interpreted as a monotone response of the } \\
\text { index to Molinari 1989's sequence) } \\
\text { intuitively changes in evenness (as interpreted by Molinari 1989) }\end{array}$ & Unrelated \\
\hline Feature 13: symmetric with regard to minor and abundant species & Unrelated \\
\hline Feature 14: skewed distributions should give a lower value & \\
\hline
\end{tabular}

Continuity when adding a new, minor species. Engen (1979) also developed evenness as a concept that was meant to be as "independent" from species richness as possible (cf. Engen 1979 p. 39 and properties (c), (d) and (e), pp. 41-43). As another manifestation of this independence, properties (c) and (d) in Engen (1979) imply that community $(2 \varepsilon, 0.5-\varepsilon, 0.5-\varepsilon)$ should have an evenness that tends to the maximum evenness when $\varepsilon$ approximates zero, so that the addition of a new, minor species did not affect evenness discontinuously. It implies that, for instance, $(0.001,0.999)$ may be more even than $(0.01$, 0.99) (cf. Engen 1979, pp. 41-42). Although Engen (1979) writes: "it is the author's view that there is no 'common sense' idea of equitability that leads to a particular ordering" of these two communities, we argue that, if we think of evenness as representing the relative apportionment of abundance among the proportion of species, then $(0.001,0.999)$ must be less even than $(0.01,0.99)$, as 
Figure 2. Lorenz curves of the minimum evenness communities comprising $2,3,5,10$ or 15 species.

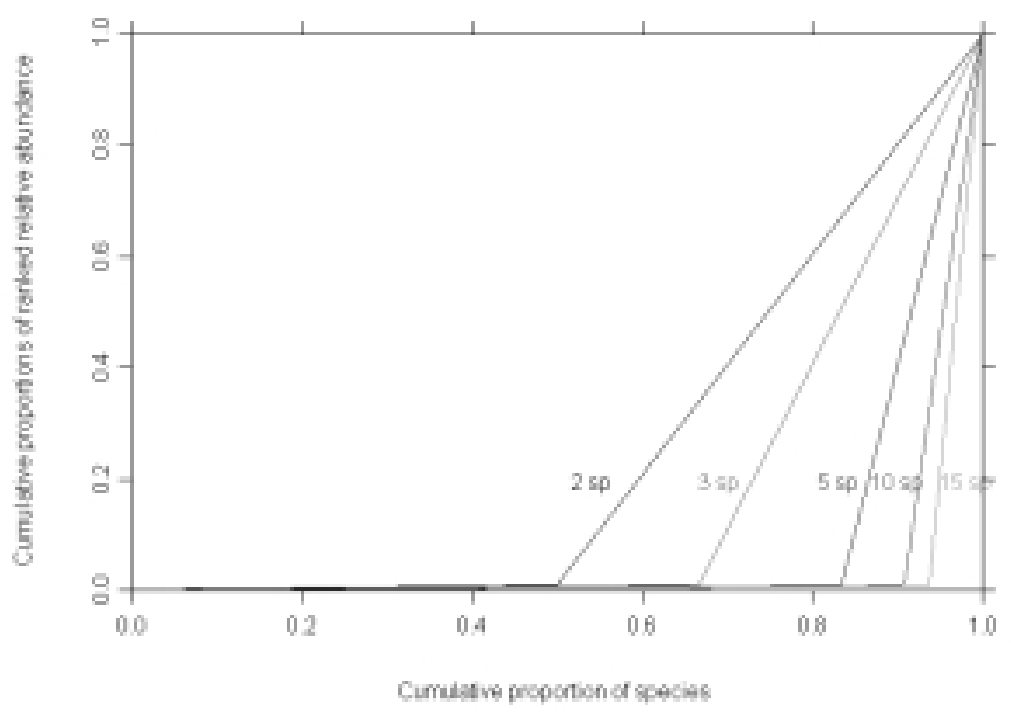

stated in the transfer principle of the Lorenz partial order. As implicitly pointed out by Engen (1979, p. 41), provided we require that maximum evenness does not depend on species richness, we have to choose between the fulfilment of the transfer principle and the continuity of evenness indices when the relative abundance of a species tends to zero and the species disappears. We choose the first. This in particular means that $(0.02,0.49,0.49)$ has an evenness that is much less than that of community $(0.5$, 0.5) (cf. also Requirement 3 in Smith and Wilson 1996, and the discussion in Smith and Wilson 1996, p. 80). A similar reasoning should apply to property (e) in Engen (1979).

\section{Evenness indices and their compatibility with the Lorenz partial order}

Lorenz curves provide an easy way to compare the intrinsic evenness of two or three communities. But when it comes to the comparison of a higher number of communities, the use of a unique number per community is easier: this is the role of evenness indices. I shall say that such an index is Lorenz-compatible (or compatible with the Lorenz partial order) if, for communities B and $\mathrm{C}$ such that $\mathrm{B}$ is intrinsically less even than $\mathrm{C}$, the index of evenness of $\mathrm{B}$ is lower than that of $\mathrm{C}$. We say that an index is weakly Lorenz-compatible if, for communities $\mathrm{B}$ and $\mathrm{C}$ such that $B$ is intrinsically less even than $C$, the index of evenness of $\mathrm{B}$ is not higher than that of $\mathrm{C}$. Previous publications have specified a list of evenness indices that are Lorenz-compatible or not (see Table 2 with my additions).

\section{Further evenness indices based on Lorenz curves}

I propose four alternative families of evenness indices compatible with the Lorenz partial order, meant to fit the formalism of Lorenz curves directly.

The first such family is $E_{G, a, b}$ the area beneath the Lorenz curve but only for a portion of the values of the abscissae $(a<x<b$, with $\{a ; b\} \in[0,1]$; cf. Fig. 3$)$. It can be considered as the restriction of the Gini index divided by 2 (cf. Table 2) to a particular region of the $\mathrm{x}$-axis. It therefore quantifies both the variation of the cumulative relative abundance in this region of the $x$-axis, and the value of cumulative relative abundance at $x=a$. The formula to calculate this index is:

$$
\begin{gathered}
\sum_{i=1}^{I(a S)+1} \frac{p_{i}}{S}(b S-a S)+\sum_{i=I(a S)+2}^{I(b S)} \frac{p_{i}}{S}\left(b S-i+\frac{1}{2}\right)- \\
\frac{p_{I(a S)+1}}{2 S}(I(a S)+1-a S)^{2}+\frac{p_{I(b S)+1}}{2 S}(I(b S)+1-b S)^{2}
\end{gathered}
$$

where $\underline{I}(x)$ denotes the integer part of the real number $x$, i.e., the closest integer to $x$ such that it is smaller than or equal to $x$.

The second family of evenness indices proposed here is even simpler: for a number $a$ between 0 and 1, it consists in determining the proportion of species necessary to obtain $a$ as cumulative relative abundance, when considering species from the most to the least abundant (cf. Fig. 4). The formula for this other index is:

$$
E_{R A, a}=1-\frac{i_{1}}{S}-\frac{1-a-\sum_{i=1}^{i_{1}} p_{i}}{S p_{i_{1}+1}},
$$




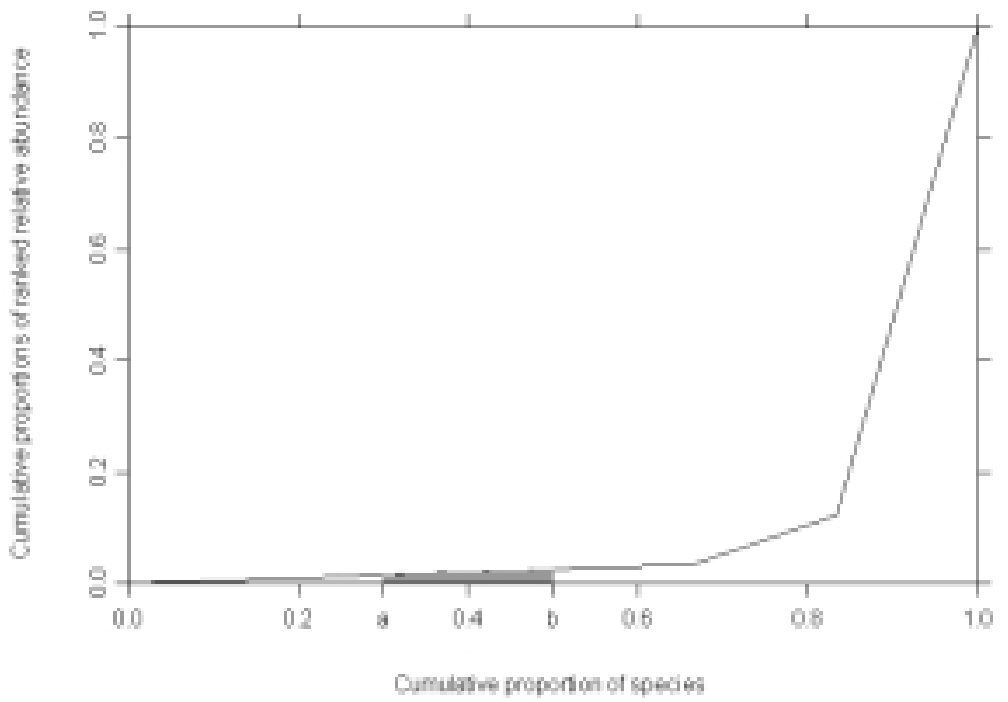

Figure 3. Schematic representation of the index $E_{G, a, b}$ for the community with abundance $(1,1,1,1$, 10, 100), $a=0.3$ and $b=$ $0.5: E_{G, a, b}$ is the area of the filled polygon, which upper limit is the Lorenz curve of the community.

where $i_{1}$ is determined iteratively, such that

$i_{1} \in\{0 ; 1 ; \ldots ; S\}, \quad \sum_{i=1}^{i_{1}} p_{i} \leq 1-a$ and $\sum_{i=1}^{i_{1}+1} p_{i}>1-a$

The third family of simple evenness indices is the converse of the previous one, since it calculates for each $a$ falling between 0 and 1 the cumulative proportion of relative abundance contributed by the fraction $a$ of the least abundant species in the community (cf. Fig. 5):

$$
E_{R S, a}=\sum_{i=1}^{I(a S)} p_{i}+p_{I(a S)+1}[a S-I(a S)] .
$$

Finally, the fourth new evenness index is calculated as the complement of the abscissa of the intersection between the Lorenz curve and the line linking the point $(0,1)$ to the point $(1,0)$ (cf. Fig. 6). The formula is given by

$$
E_{D}=\frac{1}{1+S p_{i_{1}+1}} \sum_{i=1}^{i_{1}}\left[p_{i}+\left(S-i_{1}\right) p_{i_{1}+1}\right]
$$

where $i_{1}$ is determined iteratively, such that

$$
i_{1} \in\{0 ; 1 ; \ldots ; \mathrm{S}\}, \quad \sum_{i=1}^{i_{1}} p_{i} \leq 1-\frac{i_{1}}{S} \text { and } \sum_{i=1}^{i_{1}+1} p_{i}>1-\frac{i_{1}+1}{S} .
$$

This index corresponds to the proportion of the least abundant species such that this proportion is equal to one minus the relative abundance they keep.

It is easy to show that these four new families of indices are weakly Lorenz-compatible. Therefore, they are a little less sensitive to changes in evenness than other Lo- renz compatible indices. These indices do not have 1 as maximum value: they actually vary between 0 and $\left(b^{2}\right.$ $\left.a^{2}\right) / 2$ for $E_{G, a, b} 0$ and $a$ for $E_{R A, a}$ and $E_{R S, \mathrm{a}}, 0$ and 0.5 for $E_{D}$, respectively. Although they could be transformed to take 1 as maximum value, I think that they would then be less intuitively understood (especially $E_{R A, a} E_{R S, \mathrm{a}}$, and $\left.E_{D}\right)$.

\section{Discussion}

The first point in this paper was the proposal to use the Lorenz partial order, rather than other proposed constructions, to define evenness in ecology. It appears that a crucial divergence between the Lorenz partial order, Smith and Wilson's (1996) work and Engen's (1979) construction is what happens when a very rare, new species is added to a community (especially to a perfectly even community), and, as a particular case, when a few very rare species coincide with a single superdominant species: Smith and Wilson (1996) then require that evenness should be close to its absolute minimum; the Lorenz order requires that evenness should be close to its minimum for this number of species, but not to its absolute minimum; while Engen (1979, properties (c) and (d)) requires that evenness be very close to its maximum. I prefer the behaviour of the Lorenz partial order on this point, if we accept the idea on which it is based, consisting in the quantification of the relative apportionment of abundance among the proportion of the species present. Other reasons why I favour the Lorenz partial order over Smith and Wilson's (1996) set of requirements and features are: (i) I strongly prefer the asymmetry between $(0.01,0.01,0.01$, $0.97)$ and $(0.01 .0 .33,0.33,0.33)$ if we accept that evenness is a measure of the way relative abundance is equally distributed (or not) between species; and (ii) Smith and 
Table 2. List of evenness indices and their compatibility with the Lorenz partial order. These indices were mainly gathered from Taillie (1979), Smith \& Wilson (1996) and Ricotta et al. (2001). Note that one to one transformations of these indices were not considered here (cf. for instance Nijssen et al. 1998, for two such transformations).

\begin{tabular}{|c|c|}
\hline Evenness index & Lorenz-compatibility \\
\hline $\begin{array}{l}\text { Hill (1973)'s evenness indices: } E_{H i, a}=\frac{D_{H i, a}}{S} \text {, where } \\
D_{H i, a}=\left(\sum_{i=1}^{S} p_{i}^{a}\right)^{1 /(1-a)} \text { is the exponential of Rényi } \\
(1961) \text { 's information indices }\end{array}$ & Lorenz-compatible (cf. Taillie 1979) \\
\hline $\begin{array}{l}\text { Other Hill (1973)'s evenness indices: } E_{H i, a, b}=\frac{D_{H i, a}}{D_{H i, b}} \\
\text { with } b>0\end{array}$ & Incompatible (cf. Taillie 1979) \\
\hline $\begin{array}{l}\text { Other evenness indices based on the Hill (1973) and Rényi } \\
\text { (1961) families in Smith \& Wilson (1996): } \frac{\log \left(D_{H i, 1}\right)}{\log (S)} \\
\frac{D_{H i, 1}-1}{S-1}, \frac{1-1 / D_{H i, 2}}{1-1 / S}, \frac{\log \left(D_{H i, 2}\right)}{\log (S)} \\
F_{H i, a, b}=\frac{D_{H i, a}-1}{D_{H i, b}-1}\end{array}$ & $\begin{array}{l}\text { Incompatible (cf. behaviour for requirement } \mathrm{n}^{\circ} 1 \text { in } \\
\text { Smith and Wilson 1996). }\end{array}$ \\
\hline Gini Index: $\left[2 \sum_{i=1}^{S}(S-i) p_{i}-1\right] / S$ & Lorenz-compatible (cf. Taillie 1979) \\
\hline $\begin{array}{l}\text { Camargo (1993)'s index of evenness: } \\
E^{\prime}=1-\sum_{i=1}^{S} \sum_{j=i+1}^{S} \frac{\left|p_{i}-p_{j}\right|}{S}\end{array}$ & $\begin{array}{l}\text { Incompatible, but } E^{\prime}-\frac{1}{S} \text { is compatible (it is the } \\
\text { Gini Index). }\end{array}$ \\
\hline $\begin{array}{l}\text { The inverse of the coefficient of variation of abundance: } \\
\frac{1}{C V}=\frac{1}{\sqrt{\sum_{i=1}^{S}\left(p_{i}-1 / S\right)^{2}}}\end{array}$ & $\begin{array}{l}\text { Lorenz-compatible (cf. Nijssenn et al. } 1998 \text { and } \\
\text { also Taillie } 1979 \text { pp. 56-57) }\end{array}$ \\
\hline Bulla (1994)'s index of evenness : $O=\sum_{i=1}^{S} \min \left(p_{i} ; \frac{1}{S}\right)$ & Weakly Lorenz-compatible \\
\hline Bulla (1994)'s second index of evenness : $E=\frac{O-1 / S}{1-1 / S}$ & Incompatible (not constant vis-à-vis replication) \\
\hline $\begin{array}{l}\text { Taillie (1979)'s ratios of the geometric to the algebraic } \\
\text { means of abundance } \\
G M / A M=S \exp \left[\frac{1}{S} \sum_{i=1}^{S} \log \left(p_{i}\right)\right] \text { and of the } \\
\text { harmonic to the algebraic means } \\
H M / A M=S^{2} \frac{1}{\sum_{i=1}^{S} \frac{1}{p_{i}}}\end{array}$ & $\begin{array}{l}\text { Weakly Lorenz-compatible (Lorenz-compatible } \\
\text { according to Taillie } 1979, \text { but only weakly } \\
\text { compatible when considering communities with } \\
\text { some species present but with abundance } 0 \text { ). }\end{array}$ \\
\hline
\end{tabular}


Table 2. (continued)

\begin{tabular}{|c|c|}
\hline $\begin{array}{l}\text { Taillie (1979)'s evenness indices calculated from the least } \\
\text { squares fit of gamma and lognormal models to the Lorenz } \\
\text { curve of the community. }\end{array}$ & $\begin{array}{l}\text { Lorenz-compatible (proved by considering directly } \\
\text { the least-squares formula of two communities). }\end{array}$ \\
\hline $\begin{array}{l}\text { Smith \& Wilson (1996)'s evenness index: } \\
E_{\text {var }}=1-\frac{2}{\pi} \arctan \left\{\frac{\sum_{i=1}^{S}\left(\ln \left(p_{i}\right)-\sum_{j=1}^{S} \ln \left(p_{j}\right) / S\right)^{2}}{S}\right\}\end{array}$ & $\begin{array}{l}\text { Incompatible (not systematically in agreement with } \\
\text { the transfer principle ; shown by taking derivates) }\end{array}$ \\
\hline $\begin{array}{l}\text { Smith \& Wilson (1996)'s second evenness index: } \\
E_{Q}=-\frac{2}{\pi} \arctan \left\{\frac{\sum_{i=1}^{S} \log \left(p_{i}\right) i / S}{\sum_{i=1}^{S}(i / S)^{2}}\right\} \text {, which } \\
\text { represents the arc tan transformation of the slope of the } \\
\text { scaled rank of abundance on log abundance, fitted by } \\
\text { least-squares regression }\end{array}$ & $\begin{array}{l}\text { Incompatible (not systematically in agreement with } \\
\text { the transfer principle ; shown by taking derivates) }\end{array}$ \\
\hline $\begin{array}{l}\frac{1}{V}=\frac{1}{\sum_{i=1}^{S} p_{i}\left(\ln \left(p_{i}\right)\right)^{2}-\left(\sum_{j=1}^{S} p_{j} \ln \left(p_{j}\right)\right)^{2}}\end{array}$ & Incompatible (cf. Taillie 1979) \\
\hline
\end{tabular}

Wilson's (1996) construct is close to incorporate requirements and features that are contradictory one with the other: indeed, their Requirements 1 to 3 might suffice, if stated more generally, to re-create the Lorenz partial order entirely; yet, we have seen that their Features 8, 10, 13 and 14 are in contradiction with the Lorenz partial order (cf. Table 1).

However, two limits of this partial order when related to our intuitive views on evenness need to be recognized: (i) the addition of a new, very rare species makes evenness fall "substantially" (i.e., not continuously); this is in agreement with Smith and Wilson (1996), but not with Engen (1979); and (ii) although there are some weakly Lorenz-compatible evenness indices that do have a minimum value that is independent from species richness (such as $G M / A M$ and $H M / A M$ in Table 2; and $E_{R S, a}$ above, with $a>0.5$ ), the minimum value attainable by an evenness index compatible with the Lorenz partial order should generally decrease with species richness (this stems from Fig. 2).
These two properties should make evenness vary with species richness, and therefore sampling intensity and scale. Indeed, we must distinguish between two kinds of dependency of evenness on species richness:

- evenness is independent from species richness when species richness is changed by replicating communities as in the replication principle of the Lorenz partial order (and as in Requirement 1 of Smith and Wilson 1996);

- evenness should depend on species richness when species richness is changed by incorporating or subtracting mostly relatively minor species from the community (for instance, according to a geometric distribution of abundance in the community; cf. Routledge 1983, p. 150, Tokeshi 1993). This ecological situation is close to the empirical Model (c) in Figure 1 in Wilson et al. (1999). Evenness should then decrease with species richness.

Contrary to Smith and Wilson (1996, p. 80), I therefore clearly state that this is not because evenness and species richness are independent on a theoretical point of view 
Figure 4. Schematic representation of the index $E_{R A, a}$ for the community with abundances $(1,1,1,1,10,100)$ and $a=0.9$, as related to the Lorenz curve.
Figure 5. Schematic representation of the index $E_{R S, a}$ for the community with abundances $(1,1,1,1,10,100)$ and $a=0.79$, as deduced from the Lorenz curve of the community.
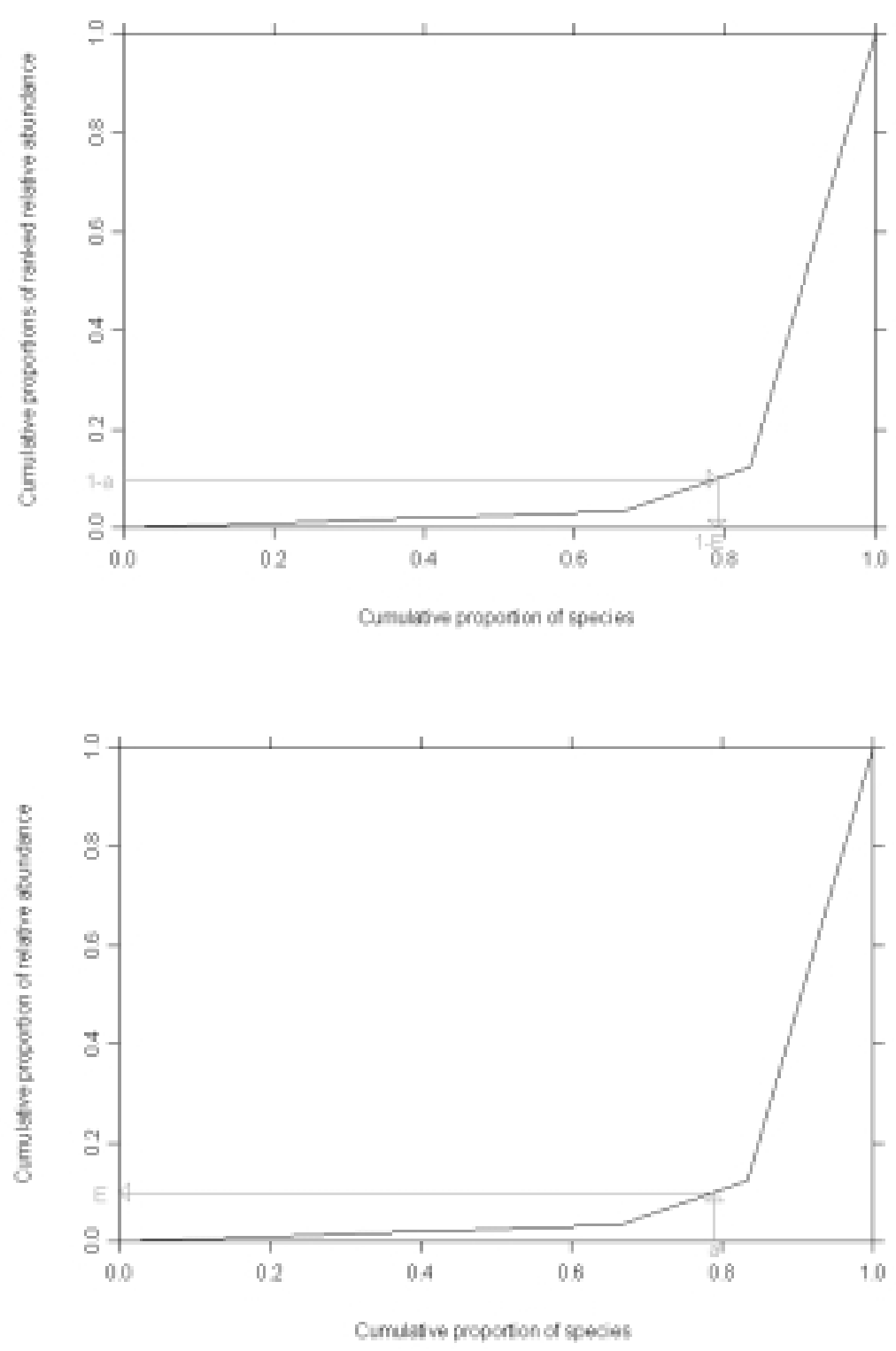

(replication principle), that they will be independent in practical ecological cases. This is not to be considered as a weakness, but as a normal consequence of the logical grounds on which evenness is based: we cannot simultaneously build an index whose maximum, minimum and value for communities of fixed abundance distribution are independent of species richness. Ways to correct this dependency of evenness on species richness will be dealt with in another paper.

These statements are valid as long as we require the transfer and the replication principles, which are at the core of the Lorenz partial order. An alternative suggested by Routledge (1983) would be to construct a different notion of equitability, more or less such that the transfer principle be valid, as well as the continuity of evenness when we add a new, very minor species. It was implicitly shown by Engen (1979, p. 41) to be incompatible with the maximum of evenness being independent on species richness. If we required only the transfer principle and the continuity property, as implicitly proposed by Routledge (1983), we should actually go back to the notion of diversity, as related to the $k$-dominance or intrinsic diversity concept (cf. Patil and Taillie 1982 and Rousseau et al. 1999).

This paper was an attempt to show that "intuitive" appraisals of evenness are not always compatible with the mathematical constructions proposed for evenness, and actually that collections of "intuitive" views of evenness may lead to an inconsistent notion. As we have shown above, this is the case for Features 8, 10, 13 and 14 in Smith and Wilson (1996). Another such example is the third graph in Figure 5 of Weiher and Keddy (1999): 

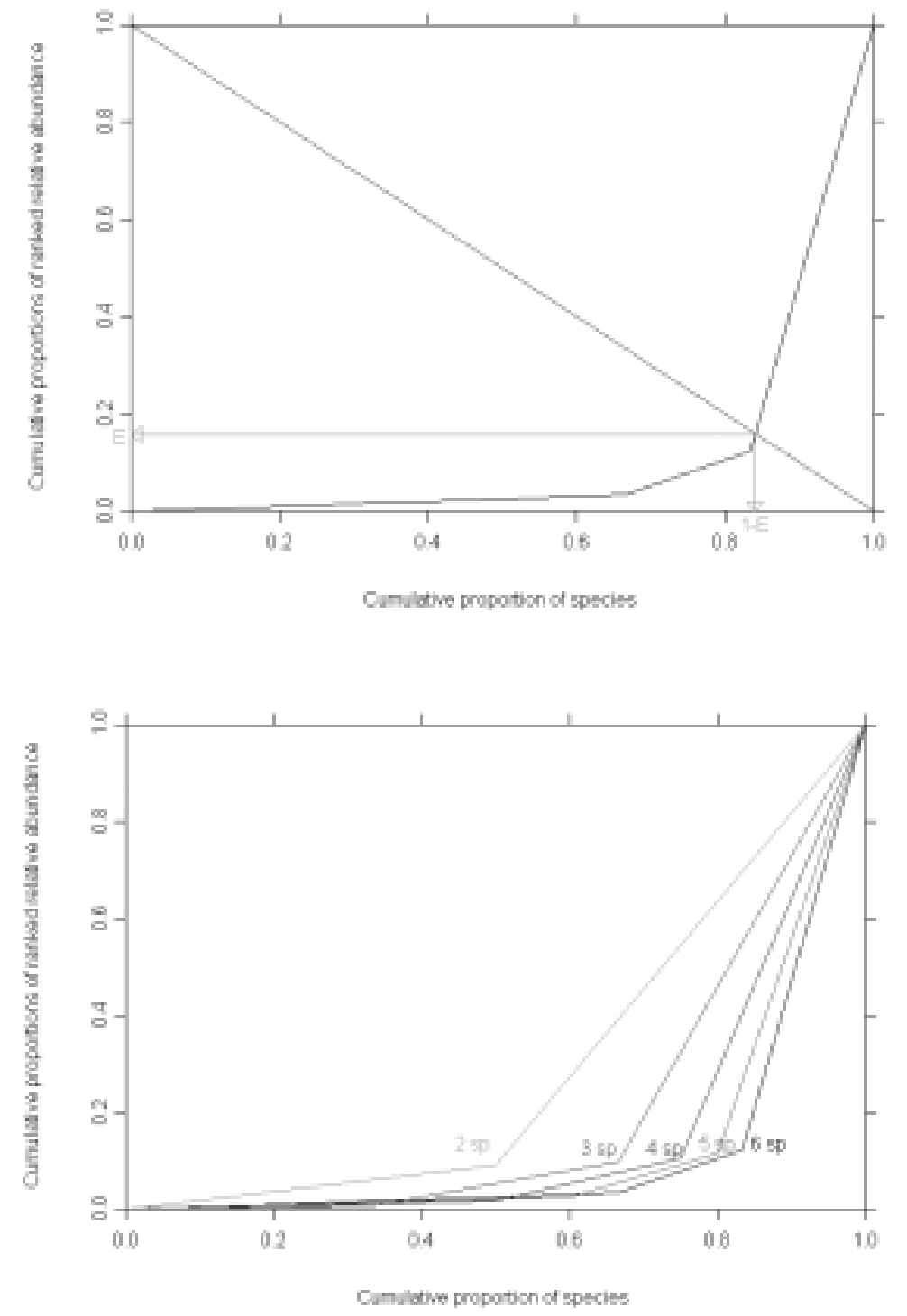

Figure 6. Schematic representation of the index $E_{D}$ for the community with abundance vector $(1,1,1,1,10,100)$.
Figure 7. Lorenz curves for the communities in the third graph of Figure 5 in Weiher and Keddy (1999), with species richness varying from 2 to 6 species. The communities are all composed of one species with abundance 100, another with abundance 10 and the remaining species all have abundance 1. A slight modification of these abundances (not in contradiction with Weiher and Keddy 1999's intuitive view) would ensure that intrinsic evenness decreases with species richness. while the authors state that evenness should increase with species number in this case (with no reason given for this statement), the Lorenz graphs of the various communities (slightly modified) show the contrary (cf. Fig. 7). In the line with Nijssen et al. (1998) and Rousseau (1999), we therefore insist that we must be very careful when gauging evenness on intuitive ideas. I also contradict the view by Engen (1979), that "it is the practical experimentation, not the philosophy, that can tell" [which properties evenness indices should meet] (p. 42). I argue that this concept must be defined clearly and uniquely, before practical experimenters (or others) build concepts of evenness based on other premises, which is of course welcome.

The use of the Lorenz partial order leads us to choose particular indices, and to deny the use of others, as even- ness indices (cf. Table 2). It is stressed that special care should be paid to this point, since the ecological literature abounds with so-called evenness indices which do not conform to the Lorenz partial order, and which are sometimes diversity indices. Especially, three of the four indices recommended by Smith and Wilson (1996) are not compatible with the Lorenz partial order; these include their two new indices. Another instance is the graphical representation of species richness, diversity and evenness of communities on the same graph, proposed by Liu (1995): it should be transformed so that the ordinate is a Hill number and the abscissa is species richness. Therefore the reader is encouraged to refer to Table 2 (or to the second part of this article) to choose a "true" evenness index. 
Of course, this is not to say that Lorenz compatibility is the only criterion to choose evenness indices. Other criteria are, for instance, unbiasedness relative to the whole community (cf. Routledge 1979 and Lande 1996, for diversity indices), sensitivity to rare and dominant species (cf. Ricotta et al. 2001) and sensitivity to sample size (Kvalseth 1991). I think the choice in Table 2 is wide enough so as to have a sufficient choice of indices on these points.

The choice of potential evenness indices is further enriched by four other families of indices. The rationale for the proposal of the first three series of indices $\left(E_{G, a, b}\right.$ $E_{R A, a} E_{R S, a}$ ) was to be able to explicitly control the region of the Lorenz curve being focused on, while using quantities that are very simply linked to the Lorenz curve. For instance, if we wish to quantify evenness related only to the $80 \%$ least dominant species, in terms of species proportion, we can use $E_{G, 0,0.8}$ or $E_{R S, 0.8}$. If we now wish to quantify the relative number of dominant species associated with $80 \%$ of abundance, we can use $E_{R A, 0.8}$. We however insist that they do not depend on all the information of the Lorenz curve and should therefore be used with care, for instance by systematically studying $E_{R A, a}$ or $E_{R S, a}$ for two or three values of $a$ that cover the range between 0 and 1 . The advantage of these quantities is, however, to define very simple, easily understandable quantifiers of evenness.

The main point in the last evenness index I propose $\left(E_{D}\right)$ was first to realize that a quantity proposed by Godron et al. (1971) to study the sharing of relative abundance between the different species actually led directly to an evenness index. The second point is the proposal by Godron et al. (1971) that "optimal" or "equilibrium" values for $E_{D}$ would not correspond to the maximum value (0.5) but to a smaller value (around 0.2). This lends support to the construction of a notion different from evenness based on the Lorenz partial order, which is beyond the scope of this paper. Godron et al. (1971) also suggested that for "equilibrium" communities, the Lorenz curve should be symmetric relative to the line linking $(0,1)$ to $(1,0)$. Finally, we think this index is also a simple one allowing to quantify evenness in an understandable way.

Contrary to Engen (1979) and Smith and Wilson (1996), I conclude that the best known way to quantify the notion of evenness in ecology is to use the Lorenz partial order, and evenness indices that are compatible with it. This is not to say that other mathematical constructs are not possible: in particular, it might be possible to replace the replication principle by another kind of independence of evenness on species richness, which might seem bio- logically more realistic. Maybe, a probabilistic framework, more related to sampling, could also be used to define evenness. In the meantime, I recommend that the Lorenz partial order be used to define evenness in ecology. This is not to say that evenness indices should be used in any ecological situation. Indeed, diversity and evenness indices do not tell the whole story of the response of species and communities to ecological factors. They should best be used in analyses, hypotheses and/or models that also study or control species composition, and maybe also species traits (cf. e.g., Wilsey and Potvin 2000, Nijs and Roy 2000).

Acknowledgements: I thank very much Carlo Ricotta, Béla Tóthmérész, Michel Godron, Frédéric Archaux and Ronald Rousseau for their comments on the manuscript. The work was funded by the French Ministry of Environment (MATE) and ECOFOR (GIP Ecofor).

\section{References}

Bulla, L. 1994. An index of evenness and its associated diversity measure. Oikos 70: 167-171.

Camargo, J. A. 1993. Must dominance increase with the number of subordinate species in competitive interactions? J. Theoret. Biol. 161: 537-542.

Dalton, H. 1920. The measurement of the inequality of incomes. Economic J. 30: 348-361.

Drobner, U., J. Bibby, B. Smith, and J. B. Wilson. 1998. The relation between community biomass and evenness: what does community theory predict, and can these predictions be tested? Oikos 82: 295-302.

Engen, S. 1979. Some basic concepts of ecological equitability. In: J. F. Grassle, G. P. Patil, W. Smith and C.Taille (eds.), Ecological Diversity in Theory and Practice. International Co-operative Publishing House, Fairland, MD. pp. 37-50.

Gerard, R. W. 1965. Intelligence, information and education. Science 148: $762-765$

Godron, M., P. Daget, J. Poissonnet, and P. Poissonnet. 1971. Some aspects of heterogeneity in grasslands of Cantal (France). In: G. P. Patil, E. C. Pielou and W. E. Walters (eds.), Many Species Populations, Ecosystems, and Systems Analysis. Pennsylvania State University Press, University Park, PA. pp. 397-415.

Hill, M. O. 1973. Diversity and evenness: a unifying notation and its consequences. Ecology 54: 427-431

Krebs, C. J. 1999. Ecological Methodology. Benjamin/Cumings, Addison-Wesley Longman Educational Publishers, New York.

Kvalseth, T. O. 1991. Note on biological diversity, evenness, and homogeneity measures. Oikos 62: 123-127.

Lande, R. 1996. Statistics and partitioning of species diversity, and similarity among multiple communities. Oikos 76: 5-13.

Liu, Q. 1995. A model for species diversity monitoring at community level and its application. Environmental Monitoring and Assessment 34: 271-287.

Marshall, A. W., and I. Olkin. 1979. Inequalities: Theory of Majorization and its Applications. Academic Press, New York.

McIntosh, R. P. 1967. An index of diversity and the relation of certain concepts to diversity. Ecology 48: 392-404.

Molinari, J. 1989. A calibrated index for the measurement of evenness. Oikos 56: 319-323. 
Nijs, I. and J. Roy. 2000. How important are species richness, species evenness and interspecific differences to productivity? A mathematical model. Oikos 88: 57-66.

Nijssen, D., R. Rousseau and P. Van Hecke. 1998. The Lorenz curve: a graphical representation of evenness. Coenoses 13: 33-38.

Patil, G. P. and C. Taillie. 1982. Diversity as a concept and its measurement. J. Amer. Stat. Assoc. 77: 548-561.

Rényi, A. 1961. On measures of entropy and information. In: J. Neyman (ed.), 4rth Berkeley Symposium on Mathematical Statistics and Probability. University of California Press, Berkeley. pp. 547-561.

Ricotta, C., E. de Zuliani, A. Pacini and G. C. Avena. 2001. On the mutual relatedness of evenness measures. Community Ecol. 2: 51-56.

Rousseau, R. 1999. Measuring biodiversity. Acta Biotheoretica 47: $1-5$.

Rousseau, R., P. Van Hecke, D. Nijsen and J. Bogaert. 1999. The relationship between diversity profiles, evenness and species richness based on partial ordering. Environmental and Ecological Statistics 6: 211-223.
Routledge, R. D. 1979. Diversity indices: which ones are admissible? J. Theoret. Biol. 76: 503-515.

Routledge, R. D. 1983. Evenness indices: are any admissible? Oikos 40: 149-151.

Smith, B. and J. B. Wilson. 1996. A consumer's guide to evenness indices. Oikos 76: 70-82.

Taillie, C. 1979. Species equitability: a comparative approach. In: J. F. Grassle, G. P. Patil, W. Smith and C.Taille (eds.), Ecological Diversity in Theory and Practice. International Co-operative Publishing House, Fairland, MD. pp. 51-62.

Tokeshi, M. 1993. Species abundance patterns and community structure. Adv. Ecol. Res. 24: 112-186.

Weiher, E. and P. A. Keddy. 1999. Relative abundance and evenness patterns along diversity and biomass gradients. Oikos 87: 355361.

Wilsey, B. R. and C. Potvin. 2000. Biodiversity and ecosystem functioning: importance of species evenness in an old field. Ecology 81: 887-892.

Wilson, J. B., J. B. Steel, W. M. King and H. Gitay. 1999. The effect of spatial scale on evenness. J. Veg. Sci. 10: 463-468. 